\title{
Refractory period of human motor nerve fibres
}

\author{
J . K I M U R A, T. Y A M A D A, A N D R. L. R O D N I T Z K Y \\ From the Division of Clinical Electrophysiology, Department of Neurology, \\ University of Iowa, Iowa City, USA
}

SUMMARY Using a collision technique, the ulnar nerve was made refractory for a shorter distance normally covered in $0.5 \mathrm{~ms}$ or a longer distance covered in $1.5 \mathrm{~ms}$. Studying the shorter refractory segment with paired shocks of maximal intensity, the test response first appeared (more than $5 \%$ of unconditioned response) at an interstimulus interval of $1.16 \pm 0.18 \mathrm{~ms}$ (mean $\pm \mathrm{SD}$ in 20 ulnar nerves). The conduction velocity of the test impulse then was $55.3 \% \pm 19.2 \%$ of normal. Recovery in amplitude of the test response was nearly complete (more than $95 \%$ ) at $2.11 \pm 0.50 \mathrm{~ms}$, when it was conducting at a speed of $81.2 \pm 17.4 \%$ of normal. The conduction velocity recovered to a level above $95 \%$ of normal at $2.65 \pm 0.65 \mathrm{~ms}$. Whereas recovery in amplitude of the test response was unrelated to the length of the refractory segment, change in latency was greater with the longer refractory segment, although not in proportion to the distance.

After passage of an impulse, an axon becomes totally inexcitable for a fraction of a millisecond, followed by progressive recovery to prestimulus level within the ensuing few milliseconds. These two phases of decreased excitability, the absolute and relative refractory periods, have been studied in experimental animals by measuring the nerve action potentials elicited by paired stimuli (Bishop and Heinbecker, 1930; Graham, 1935; Tasaki, 1953; Hodgkin, 1967; Bergmans, 1973). In 1963, Gilliatt and Willison reported the first comprehensive study on the refractory periods of human peripheral nerves. This and subsequent human studies, however, have been limited to sensory and mixed fibres (Buchthal and Rosenfalck, 1966; Lowitzsch et al., 1973; Hopf et al., 1974; Tackmann and Lehmann, 1974; Betts et al., 1976; Hopf et al., 1976). Muscle action potentials elicited by pairs of stimuli applied to motor fibres could not be measured accurately at short interstimulus intervals because of the overlap between conditioning and test responses (Wagman and Flick, 1951).

To study motor fibres using the paired shock technique, therefore, it is necessary to block the effect of the first stimulus of the pair without affecting the second. This can be achieved by "collision" if a third stimulus is delivered to the

Address for reprint requests: Jun Kimura, MD, Chief, Division of Clinical Electrophysiology, University Hospitals, Iowa City, Iowa 52242, USA.

Accepted 18 April 1978 nerve at a point distal to the paired stimuli. Using the collision technique, we showed previously that serial changes in amplitude of the test response at increasing interstimulus intervals indicated the range of the absolute refractory periods of different human motor fibres (Kimura, 1976). We have now determined changes in latency of the test response elicited at various intervals after a conditioning impulse. The course of the relative refractory period of motor fibres was measured by changes in conduction velocity.

\section{Methods}

The ulnar nerve was stimulated by paired shocks at the axilla. A single shock was given at the wrist to block the first axillary stimulus of the pair. The compound muscle action potential was recorded by surface electrodes placed on the hypothenar eminance. A stimulator with an output impedance of $250 \mathrm{ohms}$ was coupled with a transformer. The stimulus was a slightly distorted square pulse of $0.05 \mathrm{~ms}$ duration. In preliminary experiments, the intensity of the second axillary shock was altered systematically to determine the voltage change necessary to excite the lowest threshold fibres at different times after the first shock. At near threshold, however, the results obtained were very inconsistent from one trial to the next in the same subject. Consequently, in this and previous studies (Kimura, 1976) we elected to use the same axillary 
shock intensity throughout the experiment. The first (conditioning) and second (test) shocks were identical, and of just maximal intensity. The maximal stimulus, determined by delivering single shocks in increasing intensity, varied between individuals from 200 to $350 \mathrm{~V}$. A single shock delivered at the wrist was always $50 \%$ supramaximal to ensure a complete block of the first of the paired axillary stimuli.

When paired shocks at the axilla were combined with a single shock at the wrist (Fig. 1), the antidromic impulse from the wrist was eliminated by collision with the orthodromic impulse of the conditioning axillary stimulus. Because the motor axons were now cleared of antidromic activity, the impulse of the test axillary stimulus was transmitted distally, but only if the axons were excitable after the passage of the conditioning stimulus. With this technique, it was possible to adjust the point of collision and consequently the length of the nerve segment made refractory by the con-

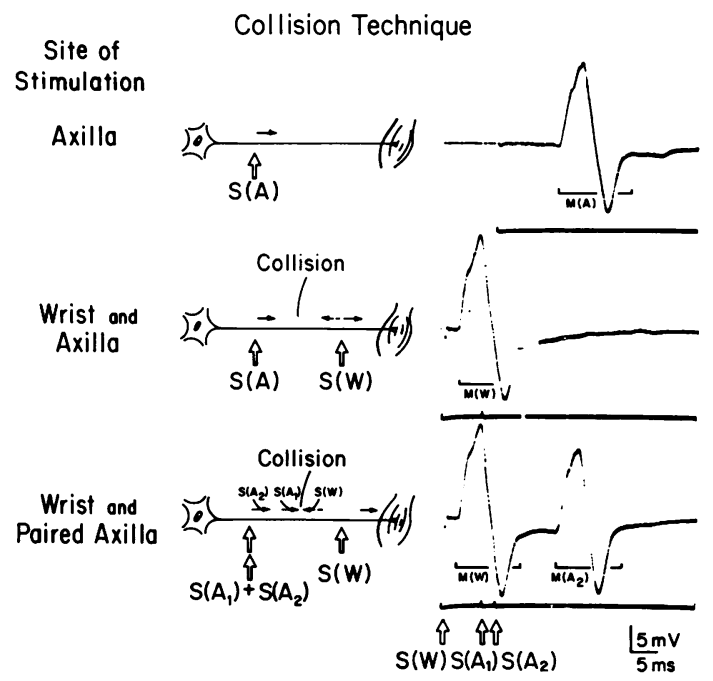

Fig. 1 Compound muscle action potentials recorded by surface electrodes placed over the abductor digiti minimi after percutaneous stimulation of the ulnar nerve. Figures on left are schematic diagrams showing orthodromic (solid arrows) and antidromic (dotted arrows) impulses. A xillary stimulation, $S(A)$, was given $6.0 \mathrm{~ms}$ after the stimulus at the wrist, $S(W)$, which triggered sweeps on the oscilloscope. With single stimulation at the wrist and the axilla (the middle tracing), the orthodromic impulse from the axilla was extinguished by collision with the antidromic impulse from the wrist. When paired shocks were delivered at the axilla (bottom tracing), $M\left(A_{2}\right)$ appeared because the first axillary stimulus, $S\left(A_{1}\right)$, cleared the path for the orthodromic impulse of the second stimulus, $S\left(A_{2}\right)$. ditioning stimulus before it was extinguished by the antidromic impulse. To compare short versus long refractory segments, two different values, 0.5 and $1.5 \mathrm{~ms}$ were chosen arbitrarily as the time of collision after delivery of the conditioning stimulus. If motor conduction velocity is assumed to be $60 \mathrm{~m} / \mathrm{s}$, therefore, the lengths of the short and long segments made refractory by the conditioning impulse travelling to the point of collision would be 3 and $9 \mathrm{~cm}$ respectively. The interval between a pair of axillary stimuli was varied from $0.1 \mathrm{~ms}$ to $4.5 \mathrm{~ms}$, in increments of $0.1 \mathrm{~ms}$ up to $2.2 \mathrm{~ms}$, then in increments of $0.2 \mathrm{~ms}$ up to $3.4 \mathrm{~ms}$, followed by 4.0 and $4.5 \mathrm{~ms}$. In some subjects, interstimulus intervals of 5.0 and $5.5 \mathrm{~ms}$ were also tested.

Although three stimuli were delivered, only two muscle potentials were recorded, because the conditioning shock given at the axilla was blocked. The two responses recorded were designated $\mathbf{M}\left(\mathrm{A}_{2}\right)$, elicited by the test axillary stimulus, and $M(W)$, evoked by the shock given at the wrist. These two potentials were clearly separate and their characteristics easily measured (Fig. 2). When the $F$ wave (recurrent discharge of anterior horn cells after antidromic invasion) was elicited by the test stimulus, it appeared at the tail end of $\mathbf{M}\left(\mathrm{A}_{2}\right)$, thus not altering its latency and affecting its amplitude very little (Kimura, 1974). The appearance of the $F$ wave in the absence of $M\left(A_{2}\right)$ was more confusing but these two potentials were easily distinguished on the basis of their latency. If a recurrent discharge was evoked by the conditioning stimulus, it could not reach the muscle because of collision with the antidromic impulse of the test stimulus.

The peak-to-peak amplitude of $\mathbf{M}\left(\mathrm{A}_{2}\right)$ was presumably proportional to the number of axons no longer refractory when the test stimulus was applied. The muscle response $\mathrm{M}(\mathrm{A})$, evoked by a single axillary shock alone, represented the total number of axons available in the nerve. Hence, the amplitude ratio, $M\left(\mathrm{~A}_{2}\right) / \mathrm{M}(\mathrm{A})$, gave the proportion of nerve fibres already conducting in response to the stimulus; that is, the degree of nerve recovery from the refractoriness induced by the conditioning shock (Fig. 3). The initial amplitude recovery of the test response was defined as a return of $\mathrm{M}\left(\mathrm{A}_{2}\right)$ to more than $5 \%$ of $\mathrm{M}(\mathrm{A})$, which was generally easier to determine than the very first, often equivocal, potential we used previously (Kimura, 1976). Full recovery was defined as the return of $\mathrm{M}\left(\mathrm{A}_{2}\right)$ to $95 \%$ of M(A) amplitude (Table).

The latency of $\mathrm{M}\left(\mathrm{A}_{2}\right)$ was measured from the stimulus artefact of the test shock to the initial 


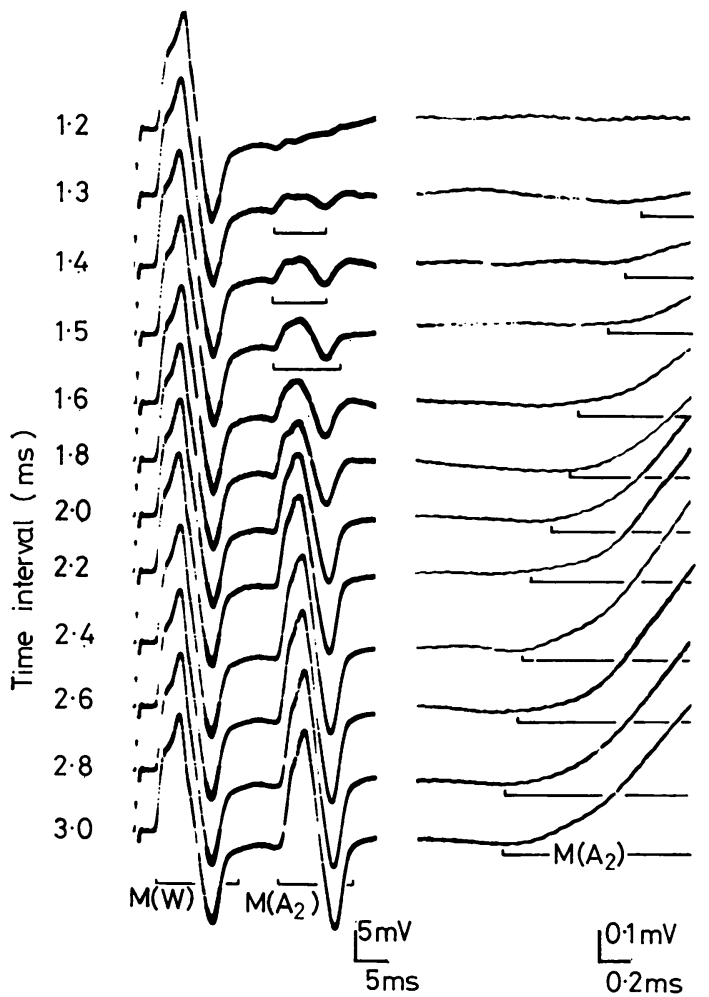

Fig. 2 Paired axillary shocks of just maximal intensity were combined with a single shock at the wrist (cf bottom tracing in Fig. 1). The first axillary stimulation, $S\left(A_{1}\right)$, was given $6.0 \mathrm{~ms}$ after the shock at the wrist, $S(W)$, so that the impulses of these two stimuli always collided $1.5 \mathrm{~ms}$ after the delivery of $S\left(A_{1}\right)$. The second axillary shock $S\left(A_{2}\right)$ was given at intervals ranging from 1.2 to $3.0 \mathrm{~ms}$ after $S\left(A_{1}\right)$. For amplitude mea:urements (left half of the figure), a slow sweep was triggered by $S(W)$. To determine latencies (right half), a fast sweep was triggered by $S\left(A_{2}\right)$ and the response displayed after a predetermined delay of $11.0 \mathrm{~ms}$.

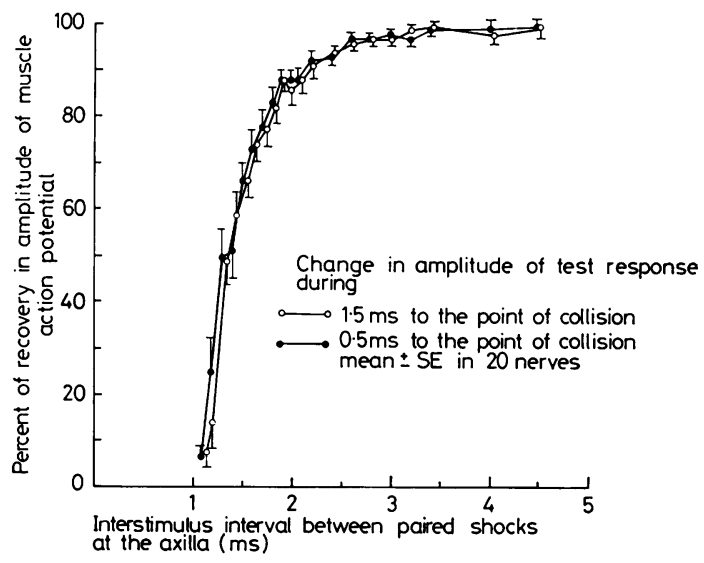

Fig. 3 The time course of recovery in amplitude of $M\left(A_{2}\right)$ after the passage of a preceding impulse in 10 healthy subjects (20 responses considering right and left sides together). The response to the second shock of the pair, $M\left(A_{2}\right)$, was converted into a percentage of the response to a single stimulus, $M(A)$, at each interstimulus interval of paired axillary stimuli. The return of $M\left(A_{2}\right)$ was practically identical whether the shorter segment normally covered in $0.5 \mathrm{~ms}$ or the longer segment normally covered in $1.5 \mathrm{~ms}$ was made refractory. The gradual increase of $M\left(A_{2}\right)$ indicates that the absolute refractory periods of the different motor fibres vary considerably, full recovery being achieved when the least excitable fibres are no longer refractory.

deflection of the evoked response, using a fast sweep triggered after a predetermined delay (Fig. 4). This value was compared to the latency of $M(A)$ evoked by a single axillary shock. Since $\mathbf{M}\left(\mathrm{A}_{2}\right)$ followed a muscle potential, $\mathrm{M}(\mathrm{W})$, elicited by the shock given at the wrist, change in neuromuscular excitability after $M(W)$ might have affected the latency of $\mathrm{M}\left(\mathrm{A}_{2}\right)$. When paired stimuli were delivered at the wrist to allow a second $\mathrm{M}(\mathrm{W})$ to appear with the same time delay as

Table Interstimulus intervals of the paired shocks and conduction velocity of the test response (mean士SD)

\begin{tabular}{|c|c|c|c|c|c|}
\hline \multirow[t]{2}{*}{$\begin{array}{l}\text { Length of refractory } \\
\text { segment }\end{array}$} & \multicolumn{2}{|c|}{$\begin{array}{l}\text { Initial recovery in amplitude } \\
\text { (test response greater than } 5 \% \text { of } \\
\text { unconditioned response) }\end{array}$} & \multicolumn{2}{|c|}{$\begin{array}{l}\text { Full recovery in amplitude } \\
\text { (test response greater than } 95 \% \text { of } \\
\text { unconditioned response) }\end{array}$} & \multirow{2}{*}{$\begin{array}{l}\text { Full recovery in } \\
\text { conduction velocity } \\
\text { (test response } \\
\text { conducts at speed } \\
\text { greater than } 95 \% \text { of } \\
\text { normal) } \\
\text { Interstimulus interval } \\
\text { between paired shocks } \\
(\text { ms) }\end{array}$} \\
\hline & $\begin{array}{l}\text { Interstimulus interval } \\
\text { between paired shocks } \\
(\mathrm{ms})\end{array}$ & $\begin{array}{l}\text { Conduction velocity } \\
\text { of test impulse } \\
(\% \text { of normal })\end{array}$ & $\begin{array}{l}\text { Interstimulus interval } \\
\text { between paired shocks } \\
(\mathrm{ms})\end{array}$ & $\begin{array}{l}\text { Conduction velocity } \\
\text { of test impulse } \\
(\% \text { of normal) }\end{array}$ & \\
\hline $\begin{array}{l}\text { Distance normally } \\
\text { covered in } 0.5 \mathrm{~ms}\end{array}$ & $1.16 \pm 0.18$ & $55.3 \pm 19.2$ & $2.11 \pm 0.50$ & $81.2 \pm 17.4$ & $2.65 \pm 0.65$ \\
\hline $\begin{array}{l}\text { Distance normally } \\
\text { covered in } 1.5 \mathrm{~ms}\end{array}$ & $1.18 \pm 0.16$ & $70.3 \pm 13.5$ & $2.16 \pm 0.52$ & $87.3 \pm 14.2$ & $2.36 \pm 0.45$ \\
\hline
\end{tabular}




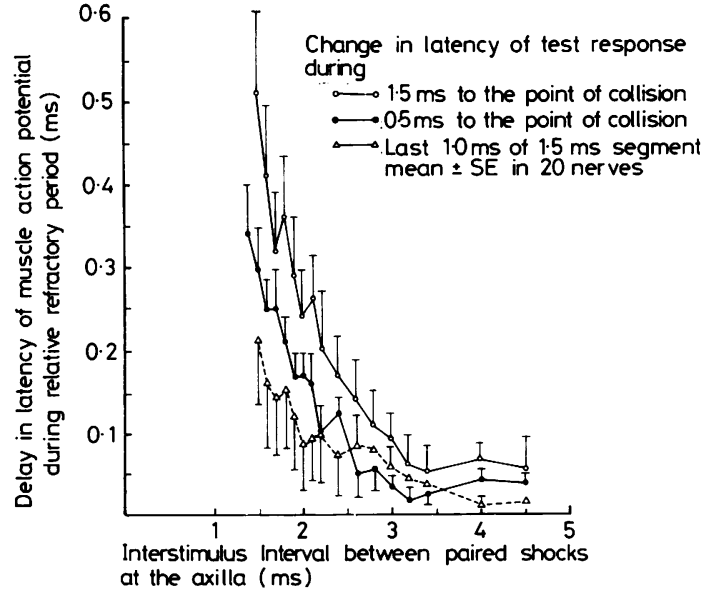

Fig. 4 The time course of recovery in latency of $M\left(A_{2}\right)$ in the same subjects as shown in Fig. 3. The latency of $M(A)$, the response to a single axillary shock, was subtracted from that of $M\left(A_{2}\right)$, the response to the second axillary shock of the pair. The recovery was significantly slower when the longer segment normally covered in $1.5 \mathrm{~ms}$ was made refractory as compared to the shorter segment normally covered in $0.5 \mathrm{~ms}$. The bottom curve (triangles) was obtained by plotting the difference between the delay that occurred during the time period of $1.5 \mathrm{~ms}$ and that during $0.5 \mathrm{~ms}$ at each interstimulus interval. The values so calculated represented the delay attributable to the last two-thirds of the longer segments, that is the distance covered during the last $1.0 \mathrm{~ms}$ of the $1.5 \mathrm{~ms}$.

$M\left(A_{2}\right)$, however, the first and second $M(W)$ were nearly identical in latency, indicating that there was no significant alteration in neuromuscular excitability (Kimura, 1976).

Because of rapid recovery of nerve excitability, changes induced by the antidromic impulse from the wrist must be small by the time the test impulse reached the point of collision. If the effect of antidromic activity on the conduction of the test impulse was considered negligible then the latency difference between $\mathrm{M}\left(\mathrm{A}_{2}\right)$ and $\mathrm{M}(\mathrm{A})$ represented a delay that occurred in the segment proximal to the point of collision. Since the time, $T$, normally required to cover the short and long refractory segment was 0.5 and $1.5 \mathrm{~ms}$ respectively, conduction velocity of the test impulse could be expressed as a percentage of normal as follows, even though the exact length of the refractory segments, D, was unknown:

$$
\begin{aligned}
& \text { Velocity of test impulse } \\
& \text { Normal conduction velocity } \times 100 \\
& =\frac{\mathrm{D} /(\mathrm{T}+\text { delay })}{\mathrm{D} / \mathrm{T}} \times 100=\frac{\mathrm{T}}{\mathrm{T}+\text { delay }} \times 100
\end{aligned}
$$

where $\mathrm{T}=0.5$ and $1.5 \mathrm{~ms}$ for the short and long refractory segments respectively.

\section{Results}

Twenty ulnar nerves were studied in 10 healthy subjects (five males) with a mean age of 30 years. In each nerve tested, the amplitude change of $\mathbf{M}\left(\mathrm{A}_{2}\right)$ obtained with shocks of maximal intensity followed a predictable time course. The amplitude recovery curves of $M\left(A_{2}\right)$ were practically identical whether the shorter or longer segment was made refractory (Fig. 3, Table).

The impulse was conducted at a slower speed than normal, if transmitted at all, during the relative refractory period (Figs. 4, 5). Slowing of conduction was greatest near the absolute refractory period, followed by progressive recovery to normal as the interstimulus interval between conditioning and test stimuli increased. When the amplitude of $\mathbf{M}\left(\mathrm{A}_{2}\right)$ recovered to $95 \%$ of that of $\mathrm{M}(\mathrm{A})$, conduction velocities were still slower than normal. With further increase in the interstimulus interval, therefore, recovery of the conduction velocity of $M\left(A_{2}\right)$ continued. The difference between the interstimulus intervals required to achieve $95 \%$ recovery in amplitude and

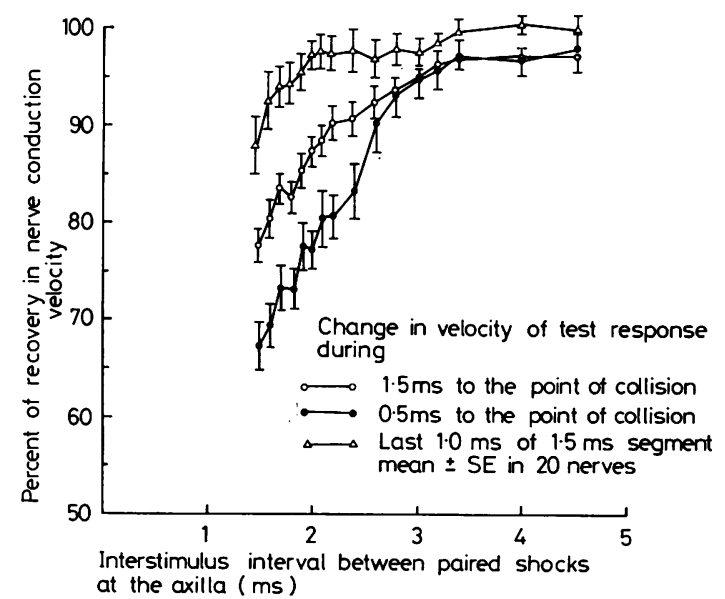

Fig. 5 The time course of recovery in conduction velocity of $M\left(A_{2}\right)$ in the same subjects as shown in Figs. 3 and 4. The conduction velocities were calculated assuming that the delay of $M\left(A_{2}\right)$ occurred primarily in the segment proximal to the point of collision, a distance normally covered in $0.5 \mathrm{~ms}$ or $1.5 \mathrm{~ms}$. In contrast to recovery in latency (cf Fig. 4) the recovery in conduction velocity was significantly faster when the refractory segment was longer. The top curve (triangles) was obtained using the calculated delay of $M\left(A_{2}\right)$ over the segment of nerve covered during the last $1.0 \mathrm{~ms}$ of the $1.5 \mathrm{~ms}$. 
those necessary for $95 \%$ recovery in conduction velocity, while present for both short and long refractory segments, was statistically significant $(\mathrm{P}<0.01)$ only for the former (Table).

Comparing two distances normally covered in 0.5 and $1.5 \mathrm{~ms}$, the delay in latency was, as expected, larger when the longer segment was made refractory. The difference between the two recovery curves (Fig. 4) was statistically significant for the interstimulus intervals of paired shocks $1.5,1.6,1.8,1.9,2.6$, and $3.0 \mathrm{~ms}(P<0.05)$. However, the delay in latency of $\mathbf{M}\left(\mathrm{A}_{2}\right)$ was not proportional to the length of the refractory segment - that is, the delay in the long segment was considerably less than three times that observed for the short segment. Indeed, the calculated delay during the last two-thirds of the longer segment, normally covered in $1.0 \mathrm{~ms}$, was about the same or less than the delay in the shorter segment normally covered in $0.5 \mathrm{~ms}$ (Fig. 4). The degree of slowing in conduction velocity must, therefore, be greater if the length of the segment made refractory by the conditioning impulse is shorter. This was indeed the case, because recovery in conduction velocity $\mathrm{M}\left(\mathrm{A}_{2}\right)$, as opposed to latency, was faster in the longer rather than the shorter refractory segment (Fig. 5). The difference was statistically significant for interstimulus intervals of $1.6,1.7,1.8,2.0 \mathrm{~ms}(\mathrm{P}<0.01)$ and $1.5,1.9,2.2$, and $2.4 \mathrm{~ms}(\mathrm{P}<0.05)$.

There was considerable variation between individual nerves in recovery of both conduction velocity and latency. Nonetheless, the course of recovery followed essentially the same expected pattern up to the $3.4 \mathrm{~ms}$ interstimulus interval. At 4.0 and $4.5 \mathrm{~ms}$, the latency of $\mathrm{M}\left(\mathrm{A}_{2}\right)$ occasionally became slightly shorter than that of $M(A)$, perhaps indicating a supernormal period of nerve excitability (Swadlow and Waxman, 1976; Waxman and Swadlow, 1976), although this was not observed universally. In some nerves tested at 5.0 and $5.5 \mathrm{~ms}$, there was reversal of the trend at these intervals and the delay in latency became longer. The cause of this reversal at late intervals remains unclear but it may be related to a late subnormal period described in sensory and mixed nerves by Gilliatt and Willison (1963) and Hopf et al. (1976).

\section{Discussion}

Although the refractory period has been examined in human sensory and mixed fibres (Gilliatt and Willison, 1963; Buchthal and Rosenfalck, 1966; Lowitzsch et al., 1973; Hopf et al., 1974; Tackmann and Lehmann, 1974; Hopf et al., 1976), studies of motor fibres have been limited previously to experimental animals. Gilliatt and Willison (1963), recording mixed nerve action potentials of the median nerve, reported absolute refractory periods of 0.6 to $0.7 \mathrm{~ms}$. Buchthal and Rosenfalck (1966) measured a value of $0.75 \mathrm{~ms}$ in sensory fibres of the median nerve. Similar results were reported by Lowitzsch et al. (1973), Hopf et al. (1974), Tackmann and Lehmann (1974), and Betts et al. (1976), all dealing with sensory or mixed fibres. An attempt to study ulnar motor fibres with paired stimuli was made by Wagman and Flick (1951) but the results were incomplete because the test response was not clearly isolated when using short interstimulus intervals. Using a collision technique, we were able to circumvent this difficulty and document that refractory characteristics of human motor fibres are similar to those of sensory fibres, the test response gradually increasing in amplitude and decreasing in latency with increasing interstimulus intervals.

The present experiment was designed to elucidate what effect, if any, the length of the refractory segment might have on the recovery of the test response. Comparing the short and long refractory segments normally covered in 0.5 and $1.5 \mathrm{~ms}$, the amplitude recovery curves of the test response were nearly identical if the intensity of the stimuli was the same (Kimura, 1976). It is likely, therefore, that the decreased amplitude of the test response was caused by failure of nerve activation at the site of stimulation. Nerve action potentials of the individual fibres, if initially activated at all, then continue to propagate distally regardless of the length of the refractory segment. This observation is consistent with the finding in animal experiments on single nerve fibres that the amplitude of the second potential of the pair recovers to a normal level as soon as it has travelled a few centimetres (Tasaki, 1953). The gradual increase in amplitude of the test response in our study, therefore, presumably indicates the range of the absolute refractory period of different fibres, which varies considerably. The initial recovery of the response is that of the most excitable fibres; full recovery is achieved when the least excitable fibres are no longer refractory. Consequently, measurements of change in amplitude fail to reveal the characteristics of the relative refractory period.

The present experiment was also designed to establish the course of the relative refractory period of motor fibres. It is known from studies of sensory and mixed fibres that nerve conduction is slowed during the relative refractory period 
(Gilliatt and Willison, 1963; Buchthal and Rosenfalck, 1966; Lowitzsch et al., 1973; Hopf et al., 1974; Tackmann and Lehmann, 1974; Hopf et al., 1976). Since, in our experiment, the latency was measured to the initial deflection of the compound action potential, latency changes evaluated were those of the fastest conducting fibres. Assuming that the fastest fibres are most excitable (Thomas et al., 1959), the recovery course of conduction velocity delineated in this study is a measure of the relative refractory period of the most excitable fibres.

In contrast to the amplitude change, the course of recovery in conduction velocity of the test response depended on the length of the refractory segment; the longer the refractory segment, the greater the change in latency of the test response. The delay in latency, however, was not proportional to the length of the refractory segments. Indeed, the delay in conduction of the test volley traversing the first one-third of the refractory segment was comparable to that over the last two-thirds. Further, average conduction velocity was faster if the refractory segment was longer, presumably because the slowing of the test impulse during the refractory period allowed an increasing interval between conditioning and test impulses as they travelled further distally (Tasaki, 1953). An increasingly longer interval between the two impulses, in turn, led to progressive recovery of the test impulse conduction velocity. Because of this regressive process, the test impulse was conducted at a relatively normal speed by the time it reached the end of the refractory segment. This tendency was greater in the longer refractory segment than it was in the shorter segment.

Our calculation of nerve conduction velocity involves the assumption that the test volley recovers to normal velocity at the exact point of collision of the conditioning and antidromic volleys. Strictly speaking this assumption is not justified since for $2-3 \mathrm{~ms}$ after the collision, there would be several centimetres of refractory nerve on either side through which the test volley must pass. The latency increase of the test response, therefore, should be attributed in part to the refractory period induced by the antidromic volley. Consequently, actual slowing of motor conduction to the point of collision is slightly less than calculated by the equation described earlier. The error, however, must be small since the test volley, delayed during the refractory period, reaches the point of collision much later than the conditioning volley. This time interval would allow substantial, if not total, recovery of the distal segment from the refractoriness induced by the antidromic volley. Nonetheless, this factor may have contributed to the very low conduction velocity derived from the short refractory segment since the effect of antidromic activity is greater the sooner the test volley reaches the point of collision after the arrival of the conditioning volley.

Studying the human nerve as a whole, as opposed to individual nerve fibres, it is not possible to define the absolute and relative refractory period for fibres of different conduction characteristics. Furthermore, in contrast to changes in amplitude which followed a predictable course in this and previous studies (Kimura, 1976), changes in latency were rather small and varied from one nerve to another. Nonetheless, our values of absolute and relative refractory periods obtained in motor fibres are very close to those of sensory and mixed fibres previously reported by Gilliatt and Willison (1963) and others as quoted above. Our findings also indicate that full recovery in amplitude of the test response precedes full recovery of conduction velocity. This suggests that the most excitable fibres are still in the relative refractory period when the least excitable fibres are no longer absolutely refractory. Similar observations have been made by others in studying sensory and mixed human fibres (Gilliatt and Willison, 1963; Buchthal and Rosenfalck, 1966; Hopf et al., 1974).

Refractory periods of motor fibres have not been adequately tested previously because of difficulty in assessing accurately the muscle potentials elicited by paired stimuli (Wagman and Flick, 1951). However, it is advantageous in clinical testing to record compound muscle action potentials as opposed to sensory nerve potentials which may be very small and difficult to elicit, especially in diseased nerves. The collision technique described here is of value in isolating the second muscle potential which otherwise would be buried in the first. The subtraction technique used by Betts et al. (1976) for analysis of paired nerve action potentials in studying refractory periods of mixed fibres may also be applicable to muscle potentials, although it requires a computerised program. The clinical value of determining refractory periods of motor fibres has yet to be tested. Since abnormalities of the refractory period of sensory and mixed fibres have been documented to occur in neuropathies (Lowitzsch et al., 1973; Tackmann and Lehmann, 1974), however, it is reasonable to assume that in diseases of peripheral nerve similar alterations may be found in the refractory characteristics of motor fibres. 
The authors wish to thank Mr D. David Walker, MSEE, for his electrical engineering assistance, Dr Leon F. Burmeister for providing statistical analysis, and Sheila Mennen, Joanne Colter, and Karen Thompson for technical assistance.

\section{References}

Bergmans, J. (1973). Physiological observations on single human nerve fibres. In New Developments in Electromyography and Clinical Neurophysiology, Vol. 2, pp. 89-127. Edited by J. E. Desmedt. Karger: Basel.

Betts, R. P., Johnston, D. M., and Brown, B. H. (1976). Nerve fibre velocity and refractory period distribution in nerve trunks. Journal of Neurology, Neurosurgery, and Psychiatry, 39, 694-700.

Bishop, G. H., and Heinbecker, P. (1930). Differentiation of axon types in visceral nerves by means of the potential record. American Journal of Physiology, 94, 170-200.

Buchthal, F., and Rosenfalck, A. (1966). Evoked action potentials and conduction velocity in human sensory nerves. Brain Research, 3, 1-122.

Gilliatt, R. W., and Willison, R. G. (1963). The refractory and supernormal periods of the human median nerve. Journal of Neurology, Neurosurgery, and Psychiatry, 26, 136-147.

Graham, H. T. (1935). The subnormal period of nerve response. American Journal of Physiology, 111, 452-465.

Hodgkin, A. L. (1967). The Conduction of the Nervous Impulse. The Sherrington Lectures VII. Liverpool University Press: Liverpool.

Hopf, H. C., Lequesne, P., and Willison, R. G. (1974). Refractory periods and lower limiting frequencies of sensory fibres of the hand. In Studies on Neuromuscular Diseases, pp. 258-263. Edited by K. Kunze and J. E. Desmedt. Karger: Basel.

Hopf, H. C., Lowitzsch, K., and Galland, J. (1976).
Conduction velocity during the supernormal and late subnormal periods in human nerve fibres. Journal of Neurology, 211, 293-296.

Kimura, J. (1974). F wave velocity in the central segment of the median and ulnar nerves: a study in normal subjects and in patients with Charcot-MarieTooth Disease. Neurology (Minneapolis), 24, 539546.

Kimura, J. (1976). A method for estimating the refractory period of motor fibres in the human peripheral nerve. Journal of the Neurological Sciences, 28, 485-490.

Lowitzsch, K., Hopf, H. C., and Schlegel, H. J. (1973). Conduction of two or more impulses in relation to the fibre spectrum in the mixed human peripheral nerve. In New Developments in Electromyography and Clinical Neurophysiology, Vol. 2, pp. 272-278. Edited by J. E. Desmedt. Karger: Basel.

Swadlow, H. A., and Waxman, S. G. (1976). Variations in conduction velocity and excitability following single and multiple impulses of visual callosal axons in the rabbit. Experimental Neurology, 53, $128-150$.

Tackmann, W., and Lehmann, H. J. (1974). Refractory period in human sensory nerve fibres. European Neurology, 12, 277-292.

Tasaki, I. (1953). Nervous Transmission. Charles C. Thomas: Springfield, Illinois.

Thomas, P. K., Sears, T. A., and Gilliatt, R. W. (1959). The range of conduction velocity in normal motor fibres to the small muscles of the hand and foot. Journal of Neurology, Neurosurgery, and Psychiatry, 22, 175-181.

Wagman, I. H., and Flick, E. W. (1951). Relative refractory period of peripheral nerve and muscle in man. American Journal of Physiology, 167, 834.

Waxman, S. G., and Swadlow, H. A. (1976). Morphology and physiology of visual callosal axons: evidence for a supernormal period in central myelinated axons. Brain Research, 113, 179-187. 